\title{
Meeting Report: Conference on Dissolution, Bioequivalence, and Bioavailability
}

\author{
Tahseen Mirza, Ph.D. ${ }^{1}$ and Vivian A. Gray ${ }^{2}$ \\ ${ }^{1}$ Novartis Pharmaceuticals, East Hanover, NJ \\ ${ }^{2}$ V. A. Gray Consulting, Hockessin, DE
}

n January 26-27, 2004 a workshop on Dissolution, Bioequivalence and Bioavailability was held in Philadelphia, $\mathrm{PA}$, during a three punch snow storm on the east coast. In spite of the bad weather, the attendance was good and the participants brought many questions and the discussions were fruitful.

The meeting began with Tahseen Mirza, Ph.D., the conference chairperson, former USP, current Associate Director Analytical and Pharmaceutical Development, Novartis Pharmaceutical Corp, summarizing the objectives of the meeting. He emphasized the significance of establishing a clear objective before developing dissolution methods. It must be specified whether it is a test of pharmaceutical quality or a test of biopharmaceutical quality and then design the method development strategies accordingly. He identified the following ten topics and questions as the focus of the workshop.

- Setting specifications by development stage

- Better understanding of the meaning of discriminating ability

- Proper media selection

- Biorelavent media - what does biorelevance mean?

- Role and future of calibrator tablets

- Method validation by development stage

- How are we getting ready to perform dissolution of low solubility drugs?

- Novel and automated dissolution equipment

- IVIVC vs. IVIVR and challenges in IVIVC

- Proper design of BE, PK and IVIVC studies

He then led the conference with a talk on Dissolution Calibration. He gave an overview of the role of calibrator tablets and provided insight on the future trends. He described a successful calibration as the one which provides assurance that the entire system comprising of the three A's; the analyst, the apparatus and the analytical procedure are working properly. He described the evolution of calibrator tablets and emphasized that the original purpose of the calibrator tablets was to minimize the inter- and intra-laboratory variability. During the past two decades, the equipment technology and analyst training have improved significantly leading to less variability and hence less reliance on calibrator tablets. During this period, the number of tests required for passing the USP Apparatus Suitability Test has been reduced to four from the original eight. He predicted that the trend is likely to continue. He pointed out ways by which historical calibration data can be used to assess the performance of the laboratory, the individual apparatus and individual locations within the apparatus. He also proposed the need for developing a modern calibrator tablet for the modern equipment.

The next speaker was Fernando J. Muzzio, Professor, Director, Pharmaceutical Engineering, of Rutgers University. His topic was Hydrodynamic-Induced Variability in USP-2 Dissolution Testing. He described how results obtained using modern fluid mechanical tools (velocimetry experiments and Computational Fluid Dynamic simulations) demonstrate that the hydrodynamic environment in a USP-2 apparatus operated under standard conditions is highly heterogeneous. This phenomenon is demonstrated using both Prednisone calibrator tablets, as well as an OTC product. Since during the course of dissolution testing, tablets experience displacements within this region, the observed performance can depend strongly on the uncontrolled tablet trajectory. While sinkers might alleviate in part this source of uncontrolled variability, they introduce additional unknowns and delay dissolution. It can also result in a systematic bias that is both unrelated to true product performance and that decreases discriminating power of the test.

The session then turned to the topic of specifications with Vivian Gray, former USP, President, V.A. Gray Consulting, Inc, putting forth an update on industry perspective on setting dissolution specifications. She spoke about how dissolution specification, or selecting the $Q$ value and time have been under debate in the US, and reviewed proceedings of a recent FDA workshop on setting dissolution specifications. She explored the proposed harmonization of the Dissolution General Chapters in the three pharmacopeias (USP, JP, and EP). Then went on to discuss the $\mathrm{ICH}$ guidance document that relates to specification setting and the controversy that surrounding using disintegration tests instead of dissolution testing. Vivian compared the pros and 
cons of stage 1 vs. 2 testing according to industry and FDA, and pointed out a pertinent article found on the internet using this link www.dissolutiontech.com/DTresour/0203art/ DTFeb03_Art2.pdf.

Petra Loos, Manager Analytical Services, Aventis Pharmaceuticals, spoke on setting and establishing specifications for dissolution tests with different dissolution profiles. She discussed the experience and data collection gained during the development of a drug product utilizing batches in toxicological and clinical trials and batches from stability studies and how it should build the basis for the development of a suitable dissolution test method and setting the acceptance criteria. Based on this background, she discussed how to develop a meaningful dissolution test and verify dissolution test conditions and results using the Biopharmaceutics Classification system and reviewing apparatus available. She reviewed the USP acceptance criteria and did an in depth analysis of the ICH Q6A Decision Tree \#7 as guidance for how to set specifications during development, accounting for global requirements and specific properties of the product tested. Biowaivers were also mentioned.

The next speaker on specifications was Ngozi Okafo, Director, Analytical Sciences, OROS Technology, member of PhRMA Dissolution Expert Team, ALZA Corporation a J\&J Company. He presented an overview of the OROS delivery platform and associated release rate testing methods using USP type VII apparatus. The strategy of setting release rate specifications at various development stages was discussed. He explored tentative acceptance criteria, in-house limits, alert limits and the final acceptance criteria, relative to modified release-type specifications.

Changing to the area of Bioequivalence, William H. Barr, Pharm D, PhD., Exec. Dir., and Professor; Center for Drug Studies, School of Pharmacy, Virginia Commonwealth University; also VP R\&D Trident Pharmaceuticals gave a talk on tackling individual bioequivalence (IBE) by examining current status and lessons learned, including several case studies. He explored how statistical procedures to determine intra-subject variation and subject-treatment interactions (population subsets) have been flawed and have not provided generally accepted regulatory tools. He stresses how important it is to understand the physiologic basis for population subsets in order to better design BA studies and establish IVIVC. He also reviewed the current regulatory status of IBE.

The ever increasingly complicated aspects of Dissolution Method Validation were discussed in detail by Ruben Lozano, Ph D., Principal Scientist, of Bristol-Myers Squibb. He presented the best practices and guidelines implemented by BMS Analytical R\&D to standardize procedures for method validation in accordance with $\mathrm{ICH}$ requirements. The parameters for validation of a dissolution method were put forth and several areas were discussed: the degree of validation needed at a particular product development stage (i.e. IND vs. NDA); validation parameters for automated dissolution methods; and type of validation performed for dissolution testing for "food effect"studies. He also brought to the attention of the audience the new USP Dissolution General Chapter on Method Development and Validation and was proposed in Pharmacopeial Forum 2004,Vol. 30 (1).

A special challenge for dissolution method development, that of establishing dissolution methods for comparators was presented by a previous speaker, Petra Loos. She pointed out that the dissolution method development for comparators play an important role during the clinical development of a compound. Dissolution of comparators has to ensure that the biopharmaceutical behavior of the compound does not change after modification by overencapsulation. Various challenges were considered:developing a dissolution test for comparators with limited information; setting the specification(s) for a dissolution test for comparators; performing a quality assessment of the changes versus the unchanged product; and accounting for specific requirements such as viral safety when purchasing comparators.

A case study evaluating USP Second Tier Dissolution Testing with enzymes was presented by Lee Bennett, Sr. Scientist, PhRMA Dissolution Expert Team, Shalini Sounderrajan, of AstraZeneca Pharmaceuticals LP. He described how gelatin crosslinking has caused many troubles in dissolution testing. The USP Capsule Working Group has suggested the addition of enzymes in dissolution testing where crosslinking is an issue. These recommendations were investigated in their labs and problems were found. Specifically, the suggested $\mathrm{pH}$ range for each enzyme is shown to be inadequate. He did suggest possible alternative criteria and will be contacting USP with these recommendations.

Nick Toltl, Ph.D., Senior Scientist, Eli Lilly and Company, presented quantitative results obtained using the heated vacuum filtration and helium sparging methods. He provided guidelines for effective deaeration by helium sparging and described the effects of other parameters such as media volume, gas flow rates, degassing time, analyst variability, and inlet filter pore size. He discussed the effects of various helium sparge parameters. He also presented critical aspects of reaeration during a dissolution experiment.

On day two, the session was begun with Vivian Gray, a previous speaker, talking on Dissolution Regulatory GMP Issues, including a case study for preparing for a preapproval inspection. This presentation covered in detail GMP issues that are pertinent to dissolution testing, focusing on the case study. She reviewed important documentation (note- 
books, logbooks, and SOP's) and discussed sample tracking and validation. She explored developing strategies for auditing a dissolution lab and suitable approaches to managing inspections.

The session returned to the $B E$ aspects of dissolution work with Robert G. Buice, Ph.D., Head, Exploratory Clinical Research Dept., Daiichi Pharmaceutical Corporation providing an overview of best practices for BE study design and IV/IVC studies. He presented examples of waivers of Class I and potential waivers of Class II and III drugs, along with conceptual IV/IVC issues. He pointed out that failure to demonstrate $\mathrm{BE}$ is frequently attributed to unsuitable study design. He emphasized the need for designing $B E$ study to be tailored to drug products' characteristics, particularly BCS category. He suggested that the establishment of a realistic correlation between the in vitro release and PK profile is sometimes difficult and that BCS might help identify certain drug product classes for which a meaningful IV/IVC can be established. He provided examples in which correlation may be less realistic. He also presented cases in which in vitro dissolution data may or may not be predictive of in vivo behavior. Situations for which multiple sources of within-subject variability may lead to challenging design decisions were presented.

Anette Müllertz, Associated Professor, PhD, at the Danish University of Pharmaceutical Sciences gave a presentation entitled"Biorelevant Dissolution / Solubilisation Methods for Poorly Soluble Class II Drugs and Lipid Based Formulations of These Drugs." She described several biorelevant dissolution tests that were developed for poorly soluble drugs by utilizing simple and inexpensive media additives such as porcine bile extract, soybean phospholipids, 1-monoglycerides and oleic acid. By using flowthrough apparatus and danazol as model compound, she found good in vitro and in vivo correlation in fasted and fed state. She pointed out that very few tests account for the presence of solubilizing lipid digestion products (LDP) in the gastro intestinal tract in the post prandial state. She described a dynamic lipolysis model, where the solubilisation of drugs during formation of LDP has shown to be very useful in the estimation of a potential food effect of a specific drug. She explained that the information gained from this type of testing can be valuable in deciding on a formulation strategy.

John R. Crison, Ph.D., Senior Scientist, at Pfizer, Inc., followed up with a talk on developing proven strategies for using surfactants. He described that as the number of poorly soluble compounds entering drug development increases, so does the use of surfactants for dissolution testing. He discussed the appropriate concentrations levels for surfactant and pointed out the potential problems. The problems discussed were the purity of sodium lauryl sulfate, the possibility of particle aggregation, mixed micelles and over solubilization and foaming.

Michelle A. Long, PhD, Research Investigator, of Abbott Laboratories reviewed the philosophy and design of testing methods for lipid-based drug delivery systems. She explained the physical processes of emulsification and solubilization with respect to dissolution testing and in vivo performance. In addition, she presented strategies to select surfactants and ensure compatibility for designing Tier II methods for gelatin encapsulated products. She identified a logical approach to the design of dissolution methods for lipid based formulations.

Wantanee Phuapradit, Research Leader, of HoffmannLa Roche presented a detailed case study of the development of a suitable high dose oral pharmaceutical dosage form and scalable manufacturing process to address technical issues for an amorphous drug with a tendency to gel in the presence of water. She described a"hot melt (nonsolvent granulation" process using Poloxamer $188, \mathrm{NF}$ as the key excipient. This water soluble, non-ionic synthetic block copolymer of ethylene oxide an propylene oxide acted as a binder to form granulation and increased the dissolution rate of the drug.

The session turned to an emphasis on instrumentation with John McKay, Ph.D., Director, Marketing, Waters Corporation describing an automated and fully integrated liquid chromatograph/photo-diode array detector/mass spectrometer. He presented several examples in which this instrument was capable of on line identification of degradation products in complex dissolution medium. The mass spectrometer can also be used as an independent piece of equipment.

Kevin Bynum, Principal Scientist, Analytical Development, of Purdue Pharma LP, presented a case study of applying and validating a fiber optic dissolution used for the investigation of the in-vitro release of solid dosage forms. This talk presented data from a number of pharmaceutical dosage forms. The application of the technique to a microgram level formulation was discussed and the application of ATR probes to dissolution testing was also examined. He compared the use of a"probe in shaft" sampling technique to more traditional sampling mechanisms. Method development optimization and validation using the fiber optic system was covered in detail.

Yihong Qiu, Formulation Development Center, of Global Pharmaceutical R\&D, Abbott Laboratories presented a brief overview of in vitro tests and factors that affect dosage form behavior and drug release/absorption in the Gl tract. A case study describing an investigation based on statistically designed experiments was presented. He described how this study led to the identification of a predictive in vitro test method for a once-daily ER dosage form and subsequent applications. 
Walter S. Woltosz, Chairman and CEO of Simulations

Plus, Inc., described a state-of-the-art simulation and modeling tool which provides a practical means to under-

stand complex interactions for many drugs. This simulation program allows predicting the likelihood of achieving $B E$ with new formulations, testing the sensitivity of PK/PD responses to likely variations in dissolution/release rate, and determining optimum in vivo release profiles to achieve specific PK/PD goals. He provided strategies to identify the most likely problem areas for a particular drug/formulation. He gave an example estimating the actual in vivo release profile for a controlled release dosage form.

Dr.Jason Liao, Sr. Biometrician, of Merck Research Laboratories presented techniques for the detection of outliers in PK, BA/BE studies supported by examples. He pointed out that one of the problems encountered in $\mathrm{PK}$ $\mathrm{BA} / \mathrm{BE}$ studies is that the data set usually contains some either extremely large and/or small observations, i.e., outliers. He also suggested that these outliers can have a dramatic effect on the conclusion. Thus, it is vital to examine these observations. For this purpose, he described a functional linear model and applied to a real data set from a BE study. He demonstrated that using this approach, one can effectively determine the outlier subject within a "formulation" level and/or with respective to another "formulation."

Navnit Shah, Ph.D., Distinguished Research Leader, of Hoffman-La Roche presented a case study describing the development and characterization of an amorphous drug formulation for improved stability and bioavailability. He described a novel approach in which the amorphous drug with a mean particle size less than 1 micron is uniformly embedded in the ionic polymer, which is an anionic copolymer based on methacrylic acid and methyl methacrylate. The resulting matrix is called as microprecipitated bulk powder (MBP). Due to the high molecular weight and high glass transition temperature of the ionic polymers, as well as their relative insolubility in water, the polymers immobilize the drug in its amorphous form, thereby providing excellent stability. The bioavailability is enhanced because the drug is embedded as amorphous form in the nanosize in the polymer matrix.

The conference was sponsored by Waters Corp. and Delphian Technologies, Inc. and featured several vendor companies. This event was organized by the Education Services Division of Barnett International as part of their annual Dissolution/BA/BE conference series. Next year's event will take place in Philadelphia during early February. 